\title{
Wonderland of silicon photonics: an interview with Professor Michal Lipson
}

\author{
Guoqing Chang \\ Chinese Academy of Sciences, Institute of Physics, Beijing, China
}

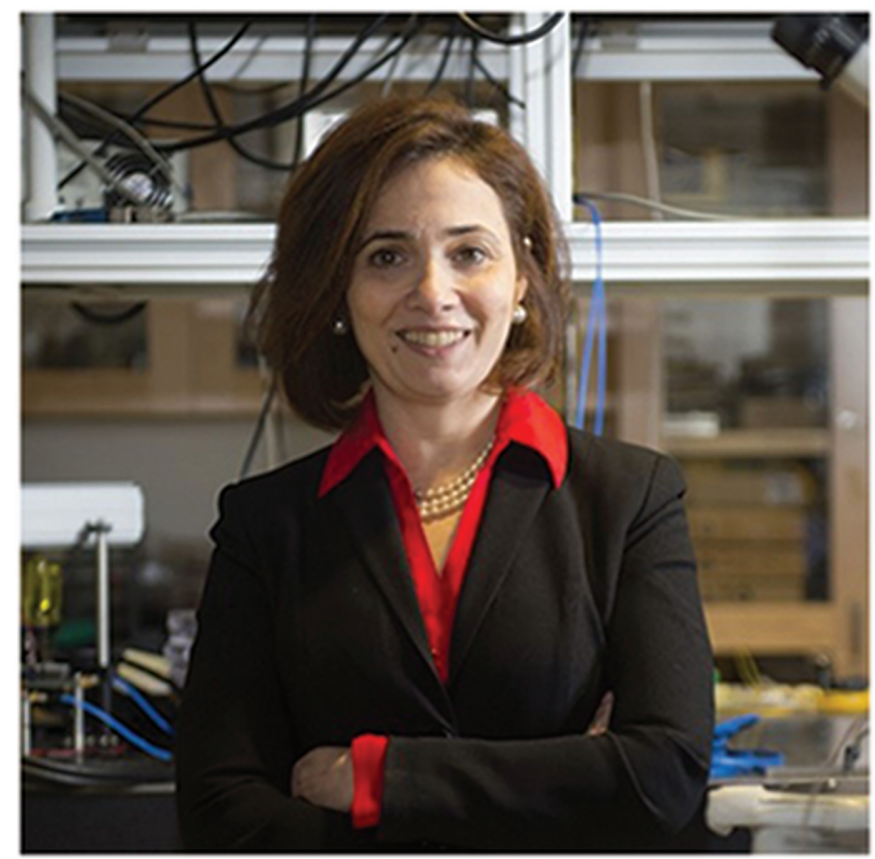

Professor Michal Lipson, Columbia University, USA

Guoqing Chang: What inspired you to choose physics as your major in college? And why did you want to pursue $\mathrm{PhD}$ ?

Michal Lipson: I was lucky to be born into a family of physicists. My father was a physicist, and my mother obtained an MS in physics. I have a twin sister, who is a professor at Boston University working in space physics. Physics was the most obvious choice for both of us since we didn't know anyone who was a physicist besides our parents. We considered for a short while other subjects such as economics or math, but my dad kept telling us physics is general enough that one can apply a lot the thinking to other areas. So we decided to study physics.

I obtained my bachelor's degree in physics in 1992. At that time, there were very few jobs for physicists. This was one of my motivation to do a $\mathrm{PhD}$. The other motivation was the fact that my degree was generalist: we studied particle physics, astronomy, solid state, etc. The more I heard about solid state, the more I loved it. So I decided to pursue a $\mathrm{PhD}$ with the focus on solid-state physics.

Guoqing Chang: Why did you choose academia as a career path? Michal Lipson: You have to remember that academia for me was not something strange: many of friends of my family were from academia. It was more natural for me to go to academia than not. That being said, I did apply to industry, both to start-ups and to larger research companies. I actually had an offer from IBM when I was looking for academic

(C) The Authors. Published by SPIE and CLP under a Creative Commons Attribution 4.0 Unported License. Distribution or reproduction of this work in whole or in part requires full attribution of the original publication, including its DOI. [DOI: 10.1117/1 AP.3.3.030501] positions. At the end I felt that my freedom to explore all kinds of ideas would be greater in academia.

Guoqing Chang: You have lived in three countries: Brazil, Israel, and now the USA. How did your experience of different cultures affect your career?

Michal Lipson: I was born in Israel and moved to Brazil when I was eight. I went back to Israel to finish my undergraduate and $\mathrm{PhD}$ degrees at the Technion-Israel Institute of Technology. In 1999, I joined the Materials Science Department of MIT as a postdoc. I joke with people that I know three languages-I speak, read, and write them fluently, but I don't know any of these languages very well. All of them are second languages for me. I don't have any preference. There is no language that I naturally gravitate to. If I' $m$ in Israel, I started thinking and speaking in Hebrew after a week; same thing happens in Brazil and in the US.

The fact that I lived in three countries with fundamentally different cultures opened my mind. I think moving from Brazil to Israel (at age 19) and doing my studies at the Technion was a wonderful experience despite facing the challenges of adapting to the difference in cultures. I'm not sure I would be where I am today career-wise if I had not moved back to Israel. Israeli people have a lot of grit-that's the strength of Israelis. The strength of Brazilians is their inclusivity: they are great collaborators.

Guoqing Chang: What was the influence of your postdoc experience at MIT on your choice of silicon photonics as a research focus when you started to work at Cornell University as an assistant professor in 2001? Michal Lipson: The history of my research started indeed with semiconductor heterostructures. I accumulated a lot of research experience by studying strong interaction between electronic excitations and cavities during my PhD. When I moved to MIT, working with Professor Lionel Kimerling, I started working on Er-doped light emitters in silicon-based structures with a focus on the strong interaction between the ion and the cavities. At that time, Professor Kimerling had a vision of silicon photonics, and I was completely taken by that vision. That's what motivated me to ask: okay, what do we need to make that reality?

Guoqing Chang: At that time, what were regarded as the important applications for silicon photonics?

Michal Lipson: I don't think energy was such a big deal at the time. It was more about the speed of interconnects. It was desired to use light to replace electronic interconnect and implement optical interconnect for clocking and high data rate. There was also a big emphasis on monolithic. Today we know that not everything really needs to be monolithic, but the general idea of transferring data using light remains the same.

Guoqing Chang: Your work on $\mathrm{GHz}$ silicon electro-optic modulator, which was published in Nature in 2005, was really a groundbreaking work. How did you come up with the idea?

Michal Lipson: In 1997, Richard Soref published a paper showing that silicon does not have an electro-optical coefficient. But he predicted what would be the effect of just plasma dispersion caused by electrons and holes in silicon and what would be the effect on the real and 
imaginary parts of the average refractive index. It was a very small effect, but not completely insignificant.

At Cornell University, we started thinking about what kind of cavity and what is the quality factor of the cavity we would need in order to use that effect. Cavities were something very natural for me, and I worked with them all my career up until then. I was excited about employing cavities to solve scientific issues. I joined Cornell in 2001, and we immediately started the research on cavities - that's when we realized that a crucial thing is to mitigate any absorption that the electron plasma causes. Finally, we turned to low-loss ring-resonator cavities to enhance the effect. Today modulators based on this effect that we have demonstrated can have extremely high performance. There is an excellent review on the topic with examples of ultrafast modulators in Advanced Photonics published recently.

Guoqing Chang: A couple of years later you started to work on on-chip frequency combs. What's the motivation behind that research?

Michal Lipson: This was in collaboration with Professor Alex Gaeta. He was interested in nonlinear silicon photonics. I remember we went to a conference presentation, and he wrote to me on a small napkin what he thought about the four-wave mixing effect in silicon. It ended up being fantastic research topic since the four-wave mixing effect given by silicon is extremely high.

Once we saw this, we understood that all the third-order nonlinear effects demonstrated in other materials will appear in silicon. But the nonlinear effects in silicon are extraordinarily higher. Another merit is the large phase matching bandwidth, which is inversely proportional to the size. So we realized that we could generate new spectral components with high efficiency pumped by a CW light (e.g., a laser pointer), and the resulting bandwidth can easily exceed an octave. We even published a paper recently to demonstrate a battery powered frequency comb.

Guoqing Chang: In 2015, you moved to Columbia University. You recently applied silicon photonics to biomedical research, such as comb optical coherence tomography (OCT), endoscopy, and brain imaging. How did you move into this research direction?

Michal Lipson: This started from a collaboration with Professor Christine Hendon in my department. They had a big challenge related to the light sources in OCT - these are traditionally inefficient and bulky. We could realize on-chip light sources very close to what they needed. So we collaborated on developing comb OCT and gradually we also applied our silicon photonics technology to other biomedical research including endoscopy and brain imaging.

Guoqing Chang: What are the current challenges faced in silicon photonics?

Michal Lipson: In my opinion, the biggest challenge in silicon photonic is the fundamental sensitivity to variation in fabrication. The variations can be as small as only 0.1 nanometer-a scale of one or two atoms, but their impact on the device performance is extremely high. I believe that smart design may solve this issue in the future. But right now, a lot of the silicon photonics is highly localized and highly confined, which leads to an extremely high sensitivity. We have to come up with ways of relaxing this sensitivity; otherwise it's very difficult to scale up the technology.

Guoqing Chang: What is your vision regarding the future of silicon photonics in the coming decade?

Michal Lipson: Silicon photonics evolved extremely fast. I never expected our work to be as impactful as it was. The applications are just tremendous and are really far-reaching. For example, who would have thought that a little chip could emit light used for LiDAR to image moving objects in 3D. New applications in microwave, computing, communication, sensing, spectroscopy, imaging, etc. are emergingdriven by the market. Of course, each application has its own challenge that needs the silicon photonics to adapt.

I love the fact that the challenges continue to emerge as well, which seems never-ending and it is a wonderful motivator for research. I believe silicon photonics will remain a wonderful field for the next decades. As long as you keep innovating you will find that your research is impactful.

Guoqing Chang: From your personal experience, how to become creative as a researcher?

Michal Lipson: As for creativity, I have a little technique that I always tell young professors: when you listen to a research talk or read a research paper, you learn about a current field and a specific work. Actually, the most important thing that you should understand is why the current field is the way it is. What is the fundamental problem in the field? Does the problem need to trace back to the Maxwell equations? Why is this a fundamental problem? Keep asking the "why." Try to avoid the influence of the masses, listen to your own voice, and understand what's really important. If the research topic you choose aligns well with what everyone else is interested in, that's great. But if not, don't worry about it. If your research topic is really important, people will follow. Never do something just because other people are working on it. Work on the topic you believe is really important. That's how to be creative.

Guoqing Guo: What is the most attractive aspect of your career as a professor?

Michal Lipson: I do feel that we are unbelievably fortunate as professors. We are paid to think creatively and to work with young talents. Every time I have a good idea, I get goose bumps on my arms; when I hear a wonderful talk given by my student or postdoc at a conference, I get goose bumps. It's an incredible gift and rewarding experience.

Guoqing Chang: What kind of advice you would like to share with $\mathrm{PhD}$ students and postdocs who are pursuing an academic career? Michal Lipson: Try to find an innovative and high-impact project you can work on with your advisor. In general, the academic job market is competitive and vibrant. All kinds of different departments, such as Chemical Engineering, Mechanical Engineering, Electrical Engineering, Applied Physics, and Materials Science, are hiring people with research experience in silicon photonics.

I would encourage people to pursue academia even if a more applied research is of interest because you can definitely do both in academiayou can carry out research and meanwhile work closely with industry.

Of course, there is also fantastic work that is being done in industrial research labs today. They are becoming more and more like some variation of the old Bell labs. Many large companies such as Facebook and Google have divisions performing cutting-edge research in silicon photonics. So that is an exciting alternative career option for researchoriented positions.

Guoqing Chang: You cofounded two companies-PicoLuz and Voyant Photonics. There is a big difference between a professor and an entrepreneur. How did you accomplish both?

Michal Lipson: The difference is not as much as you might imagine. Being a professor involves a lot of management: we manage quite a lot of funding and we manage a research group. I definitely encourage my students to start a company to commercialize our technology, and I love to get involved. It's so exciting. 
Guoqing Chang: The last question is what do you do with your spare time?

Michal Lipson: I love to spend time with my sons-one is 24 years old and the other 16 years old. During the pandemic, I enjoyed the time with them at home. I play the flute. I love reading general literature and historical biographies. I love spending time with my friends. The nice thing here at Columbia University is that we have many friends that live only a few buildings away.

Michal Lipson is the Eugene Higgins Professor of Electrical Engineering and Professor of Applied Physics at Columbia University. She completed her BS, MS, and PhD degrees in physics at the Technion-Israel Institute of Technology in 1998. In December 1998, she joined the Department of Material Science and Engineering at MIT as a postdoctoral associate. She is the inventor of over 25 patents on novel micron-size photonic structures for light manipulation and the author of over 300 papers in the major research journals in physics and optics. She is an elected member of the National Academy of Science and the American Academy for Arts and Science, and has been named by Thomson Reuters as a top $1 \%$ highly cited researcher in the field of physics. She is a recipient of the IEEE Photonics Award, the NAS Comstock Award, the OSA Wood Prize, the IEEE/OSA John Tyndall Award, the Blavatnik Award, and the Macarthur Award.

Guoqing Chang graduated with both bachelor's and master's degrees from the Electronics Engineering Department of Tsinghua University and earned his $\mathrm{PhD}$ degree in electrical engineering from the Center for Ultrafast Optical Science at the University of Michigan. After staying at the University of Michigan as a postdoctoral research fellow for about a year, he joined the Research Laboratory of Electronics at Massachusetts Institute of Technology as a postdoctoral research associate. In August 2012, he moved to the Center for Free Electron Laser (CFEL) at Hamburg (Germany) as the head of the Helmholtz Young Investigator Group "Ultrafast Laser Optics and Coherent Microscopy" under the Ultrafast Optics and X-Rays Division. He was granted tenure in 2016. In 2017, he joined the Institute of Physics, Chinese Academy of Sciences, as a professor. His current research focuses on high-power ultrafast fiber lasers, ultrafast nonlinear optics, and multi-photon microscopy for biomedical imaging. 